\title{
The efficacy of dexamethasone implants following anti- VEGF failure for macular oedema in retinal vein occlusion
}

Timothy E. Yap ${ }^{1,2}$, Salman Husein ${ }^{2}$, Juan Antonio Miralles de Imperial-Ollero ${ }^{2,3}$, Benedetta Colizzi ${ }^{2,4}$, M. Francesca Cordeiro ${ }^{1,2}$, Saad Younis ${ }^{1,2}$ *

1 The Western Eye Hospital, Imperial College Healthcare NHS Trust (ICHNT), London NW1 5QH, UK

2 The Imperial College Ophthalmic Research Group (ICORG), Imperial College London NW1 5QH, UK

3 Department of Ophthalmology, Hospital General Universitario Reina Sofía, Spain

4 Department of Ophthalmology, Università Vita-Salute San Raffaele, Milan, Italy

* Correspondence: Mr. Saad Younis, Western Eye Hospital, 153-173 Marylebone Rd, London, NW1 5QH, UK. E-mail: saad.younis@nhs.net

\section{Authors:}

1. Dr. Timothy E. Yap MBChB MA MRCP

2. Salman Husein BSc

3. Juan Antonio Miralles de Imperial-Ollero, MD

4. Benedetta Colizzi, MD

5. Prof. M. F. Cordeiro, PhD MRCP FRCOphth

6. Mr. Saad Younis, FRCSEd, DO, MBChB

\section{Site of Study:}

Western Eye Hospital, Imperial College Healthcare NHS Trust, London, UK

\section{Grants and Funds:}

Nil

\section{Data presentation:}

This paper and data has not been presented or published in its current form. A subsection of data was presented in an oral presentation at EURETINA Paris 2019.

\section{Correspondence:}

\author{
Mr. Saad Younis \\ Western Eye Hospital \\ 153-173 Marylebone Rd \\ London \\ NW1 5QH \\ UK \\ saad.younis@nhs.net
}

Declarations:

No authors have any declarations of conflict of interest relevant to the execution of this research 


\section{Keywords}

dexamethasone implant

intravitreal therapy

macular oedema

retina

retinal vein occlusion

steroid

\section{Summary Statement}

In cases of anti-VEGF failure and established vein occlusion, DEX implants are a valuable rescue treatment with large improvements in maximum VA gain compared to previous anti-VEGF treatment. Impressive structural improvements over 4 months were accompanied by more modest visual outcomes, with intraocular pressure peaking at 60 days. 


\section{Abstract}

Purpose:

To examine the efficacy of intravitreal dexamethasone implant (DEX) after antiVEGF failure in macular oedema secondary to retinal vein occlusion.

Methods:

Retrospective review of single DEX implant $(0.7 \mathrm{mg})$ after anti-VEGF failure. Local guidelines recommend switching if $\leq 5$ ETDRS letter gain and a reduction in retinal central subfield thickness of $\leq 20 \%$ after $\geq 6$ injections. Primary outcomes were peak VA gain and visual acuity (VA) at 30-days after DEX. Secondary outcomes included 15-letter gain, central subfield thickness (CST) and intraocular pressure (IOP).

Results:

62 injections in 62 patients were eligible, associated with $26 \%$ central retinal vein occlusion (CRVO) and 74\% branch retinal vein occlusion (BRVO). DEX implant significantly improved mean peak VA gain following anti-VEGF at 30 days by +13.6 letters in CRVO $(95 \% \mathrm{Cl}+1.3$ to +26.0 letters, $p<0.05)$ and +11.3 letters in BRVO $(95 \% \mathrm{Cl}+7.7$ to +15.0 letters $\mathrm{p}<0.001)$. There was a modest significant improvement in mean VA at 30 days compared to baseline $(+6$ letters, $95 \% \mathrm{Cl}+2.2$ to +9.1 letters, $\mathrm{p}<0.01$ ). IOP peaked between $30-60$ days following injection, with $31 \%$ of CRVO and $11 \%$ of BRVO patients experiencing an IOP $\geq 25 \mathrm{mmHg}$.

Conclusion:

DEX implants provide a useful rescue therapy in cases of anti-VEGF failure for macular oedema following retinal vein occlusion, resulting in improved functional outcomes at 30 days. 


\section{Introduction}

Macular oedema is a common consequence of retinal vein occlusion (RVO). This potentially sight-threatening event can also be accompanied by retinal ischaemia, neovascular glaucoma or proliferative retinopathy ${ }^{[1]}$. The 15-year incidence has been estimated at around $2 \%$ for branch retinal vein occlusion (BRVO) and $0.5 \%$ in central retinal vein occlusion (CRVO) ${ }^{[2]}$. The macular oedema itself is thought to occur due to a combination of elevated venous pressure and breakdown of the blood-retinal barrier, contributed to by release of vascular endothelial growth factor (VEGF) and other inflammatory mediators. ${ }^{[3-5]}$. Risk factors include age, hypertension, diabetes and other vascular comorbidities, as well as less common hyperviscosity syndromes and thrombophilias ${ }^{[6]}$. The advent of anti-VEGF molecules has brought about a new standard of treatment, the efficacy of which has been shown in multiple randomised controlled trials including CRUISE[7], BRAVO ${ }^{[8]} \mathrm{HORIZON}^{[9]}$, GALILEO ${ }^{[10]}$ and COPERNICUS ${ }^{[11]}$. However, a poor response to this treatment is thought to be observed in around $30 \%$ of cases ${ }^{[12]}$ following a year of treatment. Steroid therapy is often used as an important treatment option for this group of patients, however, the quantification of efficacy resulting from this switch remains relatively undefined in the literature.

Intravitreal corticosteroids in the form of triamcinolone, were first shown to improve visual outcomes in macular oedema when compared to observation alone in CRVO [13], and comparable to grid laser standard of care in BRVO ${ }^{[14]}$. This is believed to be attributable to an anti-inflammatory effect ${ }^{[15]}$ in combination with downregulation of vascular endothelial growth factor (VEGF) transcription ${ }^{[16]}$ and possible neuroprotective actions ${ }^{[17]}$. More recently, dexamethasone (DEX) has been used as a potent, water-soluble corticosteroid formulated into a biodegradable, slow-release implant in a solid polymer drug delivery system (OZURDEX, Allergan Inc., Irvine, $\mathrm{CA}$ ). The efficacy of DEX implants in macular oedema associated with RVO has been demonstrated in the GENEVA study ${ }^{[18]}$, along with the risks of raised intraocular pressure and cataract ${ }^{[19]}$.

Although multiple studies have re-assessed these beneficial effects of DEX implant both in terms of treating virgin eyes and repeat injections ${ }^{[20-23]}$, the published data for use following anti-VEGF failure is limited. Failure of anti-VEGF treatment has been demonstrated in randomised controlled trials by the frequency of rescue laser treatment $\left(20 \%\right.$ in the BRAVO study) ${ }^{[8]}$ and non-responders (18\% in CRUISE study $)^{[7,8]}$, necessitating studies such as ours. A recent meta-analysis comparing randomised controlled trials showed no significant differences in visual acuity or retinal thickness outcomes between steroid or anti-VEGF therapy used as a primary treatment ${ }^{[24]}$. Whatever the primary therapy, the visual potential in the eyes that are poorly responsive when treatment is switched remains to be fully explored. This study reports real-world data to examine the success of DEX in the setting of antiVEGF failure, to inform on the likelihood and magnitude of success and safety of switching treatments. 


\title{
Methods
}

\author{
Study design
}

A retrospective, open-label case-series of consecutive patients treated at the Western Eye Hospital, London, between October 2015 and May 2019. This study adhered to the tenets of the declaration of Helsinki and received local regulatory approval.

\section{Inclusion / exclusion criteria}

Patients were identified via an electronic patient database. Inclusion criteria included a diagnosis of macular oedema secondary to retinal vein occlusion (CRVO or BRVO), and treatment of a single dose of intravitreal dexamethasone $0.7 \mathrm{mg}$ (Ozurdex ${ }^{\circledR}$, Allergan, Inc., Irvine, CA) following failure of anti-VEGF treatment.

Anti-VEGF failure criteria included 6 consecutive anti-VEGF injections (as part of a 'treat-and-extend' or 'as-needed' protocol used in line with Royal College of Ophthalmology guidelines ${ }^{[25]}$ ), a visual response of $\leq 5$ ETDRS letters, and a CST reduction of $\leq 20 \%$.

Patients were eligible if they had baseline and follow-up VA measurements up to 6 months after treatment. Patients with multiple dexamethasone implants had only their earliest and first injection included in the analysis; only the first eye was included in bilateral cases. Hemispheric retinal vein occlusions were grouped together with $\mathrm{CRVO}^{[26]}$. Patients with a concomitant diagnosis of age-related macular degeneration, diabetic macular oedema, proliferative diabetic retinopathy, moderate to advanced glaucoma, or dense media opacity severely limiting visual potential were excluded from the study. Patients receiving DEX as part of a combined phacoemulsification procedure were also excluded, although both pseudophakic and phakic were included at the time of treatment.

\section{Visit timepoints}

Follow up visits were organised according to the routine standard of care, and retrospectively subclassified into the following time periods: '30 days' ( $\leq 45$ days), ' 60 days' (>45, $\leq 75)$, '90 days' (>75, $\leq 105)$, '120 days' (>105, $\leq 135)$, '150 days' >135, $\leq 165)$, '180 days' (>165, $\leq 195)$. Once patients had received subsequent treatment, they were excluded from all subsequent timepoints. The remaining numbers were displayed in the 'Number at Risk' tables accompanying graphs.

\section{Primary outcome}

The primary outcome was visual acuity at 30 days compared to baseline, as per previous literature ${ }^{[20]}$.

\section{Visual acuity}

The peak visual acuity response to a single DEX implant was recorded. VA measurements were either originally recorded in ETDRS letters or converted if they 
had been recorded as either logMAR or Snellen notation[27] [28]. Mean VA at each timepoint and peak VA gain 6 months following injection or prior to subsequent treatment was calculated. 5-, 10- and 15-letter visual acuity gain from baseline at any point was also assessed. Peak VA gain following the immediately preceding antiVEGF injection was calculated and compared like-for-like with DEX implant.

\section{Central subfield thickness}

Central subfield thickness was collected using Heidelberg Spectralis spectral-domain OCT (SD-OCT, Heidelberg Engineering, Heidelberg, Germany). Central subfield thickness of the macula represented the average thickness of the $1 \mathrm{~mm}$ diameter zone centred on the macula, as calculated by the in-built software. The presence of intraretinal fluid, subretinal fluid, pigmentary epithelial detachment, ellipsoid zone loss, atrophy of the retinal pigment epithelium and hyperreflective dots were noted at injection.

\section{Intraocular pressure}

Intraocular pressure was measured using a rebound tonometer (Icare, Icare Finland Oy, Vantaa). Any filtration surgery or medications to lower intraocular pressure were also noted.

\section{Statistical analysis}

Anonymised data were collated and analysed on Microsoft Excel (2019) spreadsheets. Statistical analysis was carried out using R (R Core Team, 2013). Graphs were created using ggplot ${ }^{[29]}$ and Prism (version 8.00 for Macintosh, GraphPad Software, La Jolla California USA, www.graphpad.com). Descriptive statistics for baseline characteristics used the mean and standard deviation except in the case of duration of disease, where median and range were used due to several significant outliers. To compare with baseline, a paired student's t-test was used. To compare non-parametric data, a Mann-Whitney test was performed. To compare repeated measures with baseline, a one-way ANOVA with Dunnett's correction for multiple comparisons was used. A chi-squared test was used to compare proportions. Pearson's test and ordinary least squares regression was used to analyse correlation data. Survival analysis was carried out using Kaplan-Meier survival analysis. A p-value of less than 0.05 represented statistical significance. 


\section{Results}

\section{Patient characteristics}

62 injections in 62 patients met the inclusion criteria for the study. 16 patients $(26 \%)$ with a diagnosis of CRVO and $46(74 \%)$ with BRVO. $29(47 \%)$ were male and 33 $(53 \%)$ were female. Mean age $( \pm S D)$ at the time of injection was $72.0 \pm 10.7$ years (range: 46.3 - 91.4 years). Median duration of disease pre-DEX was 2.15 yrs (range: $0.51-7.67$ years). $18(29 \%)$ patients were recorded as having type 2 diabetes mellitus, with none having type 1 diabetes mellitus. At injection, 12 (19\%) of patients were pseudophakic. 37 eyes (60\%) were dexamethasone naïve. (Table 1 )

\section{Previous anti-VEGF treatment}

The mean number of previous anti-VEGF injections $( \pm \mathrm{SD})$ was $13.6 \pm 7.5$ injections. The mean peak VA gain $( \pm S D)$ post injection was $-2.1 \pm 16.7$ letters (CRVO) and $5.6 \pm 10.0$ letters (BRVO).

\section{Baseline measures}

Mean baseline VA ( \pm SD) prior to DEX was $24.2 \pm 19.1$ letters (Snellen 3/60) in CRVO and $55.3 \pm 17.0$ (Snellen 6/24) in BRVO ( $<<0.001)$. Mean CST $( \pm$ SD) prior to DEX was $623 \pm 217 \mu \mathrm{m}$ in CRVO and $446 \pm 127$ in BRVO $(p<0.001)$. Mean IOP $( \pm$ SD) prior to DEX implant injection was $14.2 \pm 5.3 \mathrm{mmHg}$ for CRVO and $12.9 \pm 3.9$ $\mathrm{mmHg}$ for BRVO. (Table 1)

\section{Effect of DEX on visual acuity (VA)}

Overall mean VA ( \pm SD) improved from $46.2 \pm 22.1$ letters (Snellen 6/38) at baseline to $51.8 \pm 21.2$ letters (Snellen $6 / 30$ ) at the primary efficacy timepoint of 30 days $(+6$ letters, $95 \% \mathrm{Cl}+2.2$ to +9.1 letters, $\mathrm{p}<0.01$ ) (Figure 1a). $\mathrm{A} \geq 15$-letter gain in VA was achieved by 4 (25.0\%, 95\% Cl 8-53\%) CRVO patients and $11(24 \%, 95 \% \mathrm{Cl} 13-$ $39 \%)$ BRVO patients. A $\geq 10$-letter gain was achieved by 7 (44\%, 95\% Cl $21-69 \%)$ of CRVO and 17 (37.0\%, 95\% Cl 24-52\%) of BRVO patients (Figure 1d). A $\geq 15$-letter loss represented peak VA change in $1 \mathrm{CRVO}$ patient $(6 \%, 95 \% \mathrm{Cl} 16-64 \%)$ and 3 BRVO patients (7\%, 95\% Cl 2-19\%). A $\geq 10$-letter loss represented peak VA change in $1 \mathrm{CRVO}$ patient $(6 \%, 95 \% \mathrm{Cl} 0.3-32 \%)$ and $7 \mathrm{BRVO}$ patients $(15 \%, 95 \% \mathrm{Cl} 7-$ $29 \%)$. Mean peak VA improvement post-injection was $+11.6(95 \% \mathrm{Cl}+2.2$ to +20.9 letters) for CRVO and +5.5 letters $(95 \% \mathrm{Cl}+2.7$ to +8.4 letters) for BRVO. Peak VA gain was observed to be normally distributed (Figure 1c). A moderate negative correlation was observed between baseline VA and peak VA improvement after treatment in CRVO (gradient $=-0.53, p<0.05, R^{2} 0.34$ ) but not in BRVO (Figure 2). There was no correlation between VA gain and duration of disease for both CRVO and BRVO ( $p>0.05)$.

\section{Effect on central subfield thickness (CST)}

Overall mean baseline CST ( \pm SD) improved from $521 \pm 170 \mu \mathrm{m}$ (range 262 to 917 $\mu \mathrm{m})$ to $338 \pm 109 \mu \mathrm{m}$ at 30 days $(-183 \mu \mathrm{m}, 95 \% \mathrm{Cl}-257$ to $-109 \mu \mathrm{m}, \mathrm{p}<0.0001)$. Using a post-hoc ANOVA with correction for multiple comparisons, this effect was 
sustained over the 30- $(p<0.01), 60-(p<0.01)$ and 90-day $(p<0.05)$ timepoints (Figure 1b). Mean peak improvement in CST during the 6 months following DEX implant (per patient) was $-287 \mu \mathrm{m}(95 \% \mathrm{Cl}-543$ to $-31 \mu \mathrm{m})$ for CRVO and $-128(95 \% \mathrm{Cl}-173$ to $83 \mu \mathrm{m})$ for BRVO. No OCT features were associated with better or worse VA gain.

\section{Comparison with previous anti-VEGF}

There was a significant difference in peak VA improvement for CRVO $(+13.6$ letters, $95 \% \mathrm{Cl}+1.3$ to +26.0 letters, $\mathrm{p}<0.05)$ and BRVO (+11.3 letters, $95 \% \mathrm{Cl}+7.7$ to +15.0 letters, $p<0.001$ ) in favour of DEX implant when compared to previous antiVEGF injection (Table 2, Figure $3 b$ and $3 c$ ). There was no significant difference in peak CST improvement for CRVO $(-258 \mu \mathrm{m}, 95 \% \mathrm{Cl}-578 \mu \mathrm{m}$ to $+62.0 \mu \mathrm{m}, \mathrm{p}>0.05)$ in contrast to BRVO $(-124 \mu \mathrm{m}, 95 \% \mathrm{Cl}-188$ to $-60 \mu \mathrm{m}, \mathrm{p}<0.001)$ in favour of DEX implant when compared to previous anti-VEGF injection (Table 2).

Safety outcomes (IOP)

There was a significant increase in mean IOP sustained over the $30-(p<0.001)$ and 60 - day $(p<0.001)$ timepoints. The mean maximum change in IOP $( \pm S D)$ (per patient) in the 6 months following injection was $+7.4 \mathrm{mmHg}(95 \% \mathrm{Cl} 4.1-10.7 \mathrm{mmHg})$ for CRVO and $+6.7 \mathrm{mmHg}(95 \% \mathrm{Cl} 4.8-8.7 \mathrm{mmHg})$ for BRVO. Overall, the proportion of patients seen with IOP $>25 \mathrm{mmHg}$ was: $12 \%$ at 30 days $(95 \% \mathrm{Cl} 5-25 \%), 11 \%$ at 60 -days $(95 \% \mathrm{Cl} 4-28 \%), 3 \%$ at 90 days $(95 \% \mathrm{Cl} 0.2-20 \%), 6 \%$ at 120 -days $(95 \% \mathrm{Cl}$ $1-22 \%), 0$ at 150 days (95\% $\mathrm{Cl} 0-15 \%)$, and $7 \%$ at 180 days (95\% $\mathrm{Cl} 0.4-36 \%)$.

Overall, $31 \%(95 \% \mathrm{Cl} 12-59 \%)$ of CRVO and $11 \%(95 \% \mathrm{Cl} 4-24 \%)$ of BRVO patients had an IOP recorded over $25 \mathrm{mmHg}$ at any timepoint during follow up ( $p>0.05)$. No patients underwent filtration surgery to lower intraocular pressure. There were no cases of endophthalmitis related to intravitreal injection amongst the injections in the study.

\section{Survival analysis}

Survival analysis was carried out according to time until subsequent treatment was performed. There was no survival difference between vein occlusion type, diabetes status, prior anti-VEGF therapy, or early/late switching of therapy $(p>0.05)$. 


\section{Discussion}

To our knowledge, this study is the largest case-series of a single DEX implant used to treat macular oedema following retinal vein occlusion in cases of anti-VEGF treatment failure. Our results demonstrated a modest improvement in vision at 30days, with $25 \%$ of CRVO patients and $23.5 \%$ of BRVO patients achieving 15 -letter gains. When switched to a DEX implant after anti-VEGF failure, significant improvements in peak VA gain were found independent of duration of disease. Modest visual changes were accompanied by much more consistent structural improvements lasting 3 months post-injection.

Given the variable response in visual acuity (Figure 1c), it is not surprising that other studies have reported negative findings ${ }^{[22,32]}$, with ischaemia and cataract the likely culprits. Our overall finding of $24 \%$ of patients achieving a 15 -letter gain was surprisingly close to the GENEVA study ${ }^{[18]}(30 \%)$, considering the latter prospective trial included virgin eyes, excluded significantly ischaemic eyes, and contained a higher proportion of CRVO patients with lower starting VA. Similarly, the mean peak VA improvement seen in our study (7 letters) was only 3 letters less than that in the GENEVA trial. Although the GENEVA trial excluded patients based on significant ischaemia as a limiting factor, our finding of a significant correlation between baseline VA and peak VA gain demonstrates large potential gains even in those patients with a poor baseline VA. In contrast to our single-implant study, other work has examined repeated treatments\{Coscas, 2014 \#20\}, reporting 15-letter gains in $39 \%$ of patients after 2 injections, which may suggest the potential for a cumulative treatment effect.

Anti-VEGF failure is likely to be a reflection of the varying predominance of certain cytokines including VEGF, which has been correlated to levels of ischaemia ${ }^{[30,31]}$. In cases of lower comparative VEGF predominance, other inflammatory cytokines such as interleukins that steroids can regulate the expression of, may play a more important role. In comparison to other studies in anti-VEGF failure, limited published data was found ${ }^{23,33,34]}$. Georgalas et al. have recently published a prospective study of 23 patients (CRVO and BRVO) demonstrating a gain of 20 letters in CRVO and 14 letters in BRVO, however patients with baseline VA less than logMAR 1.5 (10 letters) were excluded. This would have excluded 7 of our patients, 4 of whom achieved a 15-letter gain. This suggests their method of excluding 'futile' patients may lack specificity. Alshahrani et al. published a mixed case series of macular oedema secondary to both diabetes and vein occlusion from 53 eyes ${ }^{[34]}$. For the 27 patients with RVO, their study reported a significant 17 letter gain for CRVO (1 month) and 6 letters in BRVO (3 months). Both the disease-specific timecourse and magnitude of response were not seen in our study. On comparison, their mean number of previous anti-VEGF injections was much lower (3.8 vs 13.6 injections), although having a similar time since diagnosis and starting visual acuity. This difference may suggest that continued anti-VEGF therapy may have also seen a further response or that a shorter duration of disease may have had an impact, although this association was not seen in our results. The ability to engage a wider range of molecular targets with steroid must be balanced with the difference in efficacy of treating neovascularisation on a patient-by-patient basis. 
With regards to structural changes, it is noticeable that although the majority of patients experienced structural improvement following DEX implant, this did not translate into as profound or consistent visual benefit (figure 1a). The 3-month CST reduction reported in the GENEVA study was greater than our overall mean peak CST reduction ( $\pm S D)$, as to be expected in untreated eyes $(263 \pm 217 \mu \mathrm{m}$ vs $147 \pm$ $170 \mu \mathrm{m})$. Although ischaemia is likely to be implicated in this, our study did find substantial gains in VA amongst some patients with poor starting VA as well as a moderate correlation between starting VA and maximum VA gain in CRVO(Figure 2). This suggests the magnitude of visual loss is closely related to oedema and not always proportionally matched or limited by ischaemia and disruption of the retinal architecture, especially in a patient population with a longer duration of disease such as ours.

Peak IOP rise was seen at around 60 days with $16 \%$ of patients experiencing an IOP rise of $10 \mathrm{mmHg}$ or greater in comparison to $32.8 \%$ reported in the GENEVA trial[18] and $27 \%$ of patients over $25 \mathrm{mmHg}$ found by Joshi et al[ ${ }^{[20]}$. The lower rates in our study may have been a reflection of the less stringent follow-up protocols, but also varying treatment thresholds for ocular hypertension. The higher proportion in CRVO is possibly explained by the different risk factor profile (raised IOP) and higher starting IOP in this subgroup.

The distribution of maximum responses did reveal a cluster of CRVO patients with an excellent response (Figure 1c). OCT predictors of success have been reported to include an intact inner segment-outer segment interface on OCT for visual recovery ${ }^{[35]}$ as reported by one small case series. However larger studies on this topic have focused on anti-VEGF therapy ${ }^{[6,37]}$. The question that still remains to be answered is how to predict which patients being treated with anti-VEGF therapy will benefit most from switching to steroids. However, no OCT or patient characteristics investigated in this study were able to provide this. Additionally, post-hoc analyses of the GENEVA trial showed advantages to early steroid treatment in BRVO but not in CRVO[38], an effect also shown with anti-VEGF treatment ${ }^{[39,40]}$. Again, survival analysis of our data did not demonstrate a per-treatment advantage of an early switch from anti-VEGF therapy, perhaps indicting the importance of treatment per se.

The limitations of this study are inherent to the analysis of real-world data. Some patients were not seen at each timepoint or included for a total of 6 months as further treatments were instigated and therefore were excluded from further analysis. We have attempted to transparently describe the data by quoting the remaining numbers of patients in the analysis below graphs, however also prospectively attempted to capture treatment effect by using a paired statistical test based on the efficacy timepoints of previous published work ${ }^{[20]}$, given the wide variation in visual acuities.

Given the frequency of anti-VEGF failure, we consider our data useful for making clinical decisions, counselling patients on the probability of success, and the expected improvement upon prior anti-VEGF treatment. There is a significant proportion of patients that will see substantial benefit from switching to DEX, however the only apparent way to predict these patients is a trial of treatment. The future of metabolomics and personalised medicine however, may be able to provide more specific ways to match patients to treatments, with the avoidance of exposing them to unnecessary risks. 



\section{References}

1. Karia N. Retinal vein occlusion: pathophysiology and treatment options. Clin Ophthalmol. 2010;4:809-816.

2. Klein R, Moss SE, Meuer SM, Klein BE. The 15-year cumulative incidence of retinal vein occlusion: the Beaver Dam Eye Study. Arch Ophthalmol. 2008;126(4):513-518.

3. Aiello LP, Bursell SE, Clermont A, et al. Vascular endothelial growth factor-induced retinal permeability is mediated by protein kinase $\mathrm{C}$ in vivo and suppressed by an orally effective beta-isoform-selective inhibitor. Diabetes. 1997;46(9):1473-1480.

4. Antonetti DA, Barber AJ, Hollinger LA, Wolpert EB, Gardner TW. Vascular endothelial growth factor induces rapid phosphorylation of tight junction proteins occludin and zonula occluden 1. A potential mechanism for vascular permeability in diabetic retinopathy and tumors. J Biol Chem. 1999;274(33):23463-23467.

5. Suzuki Y, Nakazawa M, Suzuki K, Yamazaki H, Miyagawa Y. Expression profiles of cytokines and chemokines in vitreous fluid in diabetic retinopathy and central retinal vein occlusion. Jpn J Ophthalmol. 2011;55(3):256-263.

6. Kolar P. Risk factors for central and branch retinal vein occlusion: a meta-analysis of published clinical data. J Ophthalmol. 2014;2014:724780.

7. Brown DM, Campochiaro PA, Singh RP, et al. Ranibizumab for macular edema following central retinal vein occlusion: six-month primary end point results of a phase III study. Ophthalmology. 2010;117(6):1124-1133 e1121.

8. Campochiaro PA, Heier JS, Feiner L, et al. Ranibizumab for macular edema following branch retinal vein occlusion: six-month primary end point results of a phase III study. Ophthalmology. 2010;117(6):1102-1112 e1101.

9. Heier JS, Campochiaro PA, Yau L, et al. Ranibizumab for macular edema due to retinal vein occlusions: long-term follow-up in the HORIZON trial. Ophthalmology. 2012;119(4):802-809.

10. Korobelnik JF, Holz FG, Roider J, et al. Intravitreal Aflibercept Injection for Macular Edema Resulting from Central Retinal Vein Occlusion: One-Year Results of the Phase 3 GALILEO Study. Ophthalmology. 2014;121(1):202-208.

11. Heier JS, Clark WL, Boyer DS, et al. Intravitreal aflibercept injection for macular edema due to central retinal vein occlusion: two-year results from the COPERNICUS study. Ophthalmology. 2014;121(7):1414-1420 e1411.

12. Menke MN, Ebneter A, Zinkernagel MS, Wolf S. Differentiation between Good and Low-Responders to Intravitreal Ranibizumab for Macular Edema Secondary to Retinal Vein Occlusion. J Ophthalmol. 2016;2016:9875741.

13. Ip MS, Scott IU, VanVeldhuisen PC, et al. A randomized trial comparing the efficacy and safety of intravitreal triamcinolone with observation to treat vision loss associated with macular edema secondary to central retinal vein occlusion: the Standard Care vs Corticosteroid for Retinal Vein Occlusion (SCORE) study report 5. Arch Ophthalmol. 2009;127(9):1101-1114.

14. Scott IU, Ip MS, VanVeldhuisen PC, et al. A randomized trial comparing the efficacy and safety of intravitreal triamcinolone with standard care to treat vision loss associated with macular Edema secondary to branch retinal vein occlusion: the Standard Care vs Corticosteroid for Retinal Vein Occlusion (SCORE) study report 6. Arch Ophthalmol. 2009;127(9):1115-1128.

15. Lee HB, Pulido JS, McCannel CA, Buettner H. Role of inflammation in retinal vein occlusion. Can J Ophthalmol. 2007;42(1):131-133. 
16. Pe'er J, Folberg R, Itin A, Gnessin H, Hemo I, Keshet E. Vascular endothelial growth factor upregulation in human central retinal vein occlusion. Ophthalmology. 1998;105(3):412-416.

17. Jeanneteau F, Garabedian MJ, Chao MV. Activation of Trk neurotrophin receptors by glucocorticoids provides a neuroprotective effect. Proc Natl Acad Sci U S A. 2008;105(12):4862-4867.

18. Haller JA, Bandello F, Belfort R, Jr., et al. Randomized, sham-controlled trial of dexamethasone intravitreal implant in patients with macular edema due to retinal vein occlusion. Ophthalmology. 2010;117(6):1134-1146 e1133.

19. Gewaily D, Muthuswamy K, Greenberg PB. Intravitreal steroids versus observation for macular edema secondary to central retinal vein occlusion. Cochrane Database Syst Rev. 2015(9):CD007324.

20. Joshi L, Yaganti S, Gemenetzi M, et al. Dexamethasone implants in retinal vein occlusion: 12-month clinical effectiveness using repeat injections as-needed. $\mathrm{Br} \mathrm{J}$ Ophthalmol. 2013;97(8):1040-1044.

21. Garweg JG, Zandi S. Retinal vein occlusion and the use of a dexamethasone intravitreal implant (Ozurdex(R)) in its treatment. Graefes Arch Clin Exp Ophthalmol. 2016;254(7):1257-1265.

22. Coscas G, Augustin A, Bandello F, et al. Retreatment with Ozurdex for macular edema secondary to retinal vein occlusion. Eur J Ophthalmol. 2014;24(1):1-9.

23. Tservakis I, Koutsandrea C, Papaconstantinou D, Paraskevopoulos T, Georgalas I. Safety and efficacy of dexamethasone intravitreal implant (Ozurdex) for the treatment of persistent macular edema secondary to retinal vein occlusion in eyes previously treated with anti-vascular endothelial growth factors. Curr Drug Saf. 2015;10(2):145151.

24. Hu Q, Li H, Xu W, Du Y, Ma C, He J. Comparison between Ozurdex and intravitreal anti-vascular endothelial growth factor treatment for retinal vein occlusion-related macular edema: A systematic review and meta-analysis of randomized controlled trials. Indian J Ophthalmol. 2019;67(11):1800-1809.

25. Sivaprasad S, Amoaku WM, Hykin P, Group RVOG. The Royal College of Ophthalmologists Guidelines on retinal vein occlusions: executive summary. Eye (Lond). 2015;29(12):1633-1638.

26. Schmidt-Erfurth U, Garcia-Arumi J, Gerendas BS, et al. Guidelines for the Management of Retinal Vein Occlusion by the European Society of Retina Specialists (EURETINA). Ophthalmologica. 2019;242(3):123-162.

27. Beck RW, Moke PS, Turpin AH, et al. A computerized method of visual acuity testing: adaptation of the early treatment of diabetic retinopathy study testing protocol. Am J Ophthalmol. 2003;135(2):194-205.

28. Schulze-Bonsel K, Feltgen N, Burau H, Hansen L, Bach M. Visual acuities "hand motion" and "counting fingers" can be quantified with the freiburg visual acuity test. Invest Ophthalmol Vis Sci. 2006;47(3):1236-1240.

29. Wickham H. ggplot2: Elegant Graphics for Data Analysis. Springer-Verlag New York; 2016.

30. Noma H, Funatsu H, Mimura T, Harino S, Hori S. Vitreous levels of interleukin-6 and vascular endothelial growth factor in macular edema with central retinal vein occlusion. Ophthalmology. 2009;116(1):87-93.

31. Noma H, Minamoto A, Funatsu H, et al. Intravitreal levels of vascular endothelial growth factor and interleukin-6 are correlated with macular edema in branch retinal vein occlusion. Graefes Arch Clin Exp Ophthalmol. 2006;244(3):309-315. 
32. Mayer WJ, Wolf A, Kernt M, et al. Twelve-month experience with Ozurdex for the treatment of macular edema associated with retinal vein occlusion. Eye (Lond). 2013;27(7):816-822.

33. Georgalas L, Tservakis I, Kiskira EE, Petrou P, Papaconstantinou D, Kanakis M. Efficacy and safety of dexamethasone intravitreal implant in patients with retinal vein occlusion resistant to anti-VEGF therapy: a 12-month prospective study. Cutan Ocul Toxicol. 2019;38(4):330-337.

34. Alshahrani ST, Dolz-Marco R, Gallego-Pinazo R, Diaz-Llopis M, Arevalo JF, Group KICRS. INTRAVITREAL DEXAMETHASONE IMPLANT FOR THE TREATMENT OF REFRACTORY MACULAR EDEMA IN RETINAL VASCULAR DISEASES: Results of the KKESH International Collaborative Retina Study Group. Retina. 2016;36(1):131-136.

35. Coscas G, Coscas F, Zucchiatti I, Glacet-Bernard A, Soubrane G, Souied E. SD-OCT pattern of retinal venous occlusion with cystoid macular edema treated with Ozurdex(R). Eur J Ophthalmol. 2011;21(5):631-636.

36. Gallego-Pinazo R, Dolz-Marco R, Pardo-Lopez D, et al. Ranibizumab for serous macular detachment in branch retinal vein occlusions. Graefes Arch Clin Exp Ophthalmol. 2013;251(1):9-14.

37. Yunoki T, Miyakoshi A, Nakamura T, Fujita K, Fuchizawa C, Hayashi A. Treatment of macular edema due to branch retinal vein occlusion with single or multiple intravitreal injections of bevacizumab. Jpn J Ophthalmol. 2012;56(2):159-164.

38. Yeh WS, Haller JA, Lanzetta P, et al. Effect of the duration of macular edema on clinical outcomes in retinal vein occlusion treated with dexamethasone intravitreal implant. Ophthalmology. 2012;119(6):1190-1198.

39. Brown DM, Heier JS, Clark WL, et al. Intravitreal aflibercept injection for macular edema secondary to central retinal vein occlusion: 1-year results from the phase 3 COPERNICUS study. Am J Ophthalmol. 2013;155(3):429-437 e427.

40. Thach AB, Yau L, Hoang C, Tuomi L. Time to clinically significant visual acuity gains after ranibizumab treatment for retinal vein occlusion: BRAVO and CRUISE trials. Ophthalmology. 2014;121(5):1059-1066. 